\title{
Differential transcriptional networks associated with key phases of ingrowth wall construction in trans-differentiating epidermal transfer cells of Vicia faba cotyledons
}

\author{
Hui-Ming Zhang ${ }^{1}$, Simon Wheeler ${ }^{1}$, Xue Xia ${ }^{1}$, Ruslana Radchuk², Hans Weber ${ }^{2}$, Christina E Offler ${ }^{1}$
} and John W Patrick ${ }^{1 *}$

\begin{abstract}
Background: Transfer cells are characterized by intricate ingrowth walls, comprising an uniform wall upon which wall ingrowths are deposited. The ingrowth wall forms a scaffold to support an amplified plasma membrane surface area enriched in membrane transporters that collectively confers transfer cells with an enhanced capacity for membrane transport at bottlenecks for apo-/symplasmic exchange of nutrients. However, the underlying molecular mechanisms regulating polarized construction of the ingrowth wall and membrane transporter profile are poorly understood.

Results: An RNAseq study of an inducible epidermal transfer cell system in cultured Vicia faba cotyledons identified transfer cell specific transcriptomes associated with uniform wall and wall ingrowth deposition. All functional groups of genes examined were expressed before and following transition to a transfer cell fate. What changed were the isoform profiles of expressed genes within functional groups. Genes encoding ethylene and $\mathrm{Ca}^{2+}$ signal generation and transduction pathways were enriched during uniform wall construction. Auxin-and reactive oxygen species-related genes dominated during wall ingrowth formation and ABA genes were evenly expressed across ingrowth wall construction. Expression of genes encoding kinesins, formins and villins was consistent with reorganization of cytoskeletal components. Uniform wall and wall ingrowth specific expression of exocyst complex components and SNAREs suggested specific patterns of exocytosis while dynamin mediated endocytotic activity was consistent with establishing wall ingrowth loci. Key regulatory genes of biosynthetic pathways for sphingolipids and sterols were expressed across ingrowth wall construction. Transfer cell specific expression of cellulose synthases was absent. Rather xyloglucan, xylan and pectin biosynthetic genes were selectively expressed during uniform wall construction. More striking was expression of genes encoding enzymes for re-modelling/degradation of cellulose, xyloglucans, pectins and callose. Extensins dominated the cohort of expressed wall structural proteins and particularly so across wall ingrowth development. Ion transporters were selectively expressed throughout ingrowth wall development along with organic nitrogen transporters and a large group of ABC transporters. Sugar transporters were less represented.
\end{abstract}

Conclusions: Pathways regulating signalling and intracellular organization were fine tuned whilst cell wall construction and membrane transporter profiles were altered substantially upon transiting to a transfer cell fate. Each phase of ingrowth wall construction was linked with unique cohorts of expressed genes.

Keywords: Transfer cell, Transcriptome, Cell wall construction, Uniform wall, Wall ingrowth, Transporters, Seed development, Vicia faba

\footnotetext{
* Correspondence: John.Patrick@newcastle.edu.au

'School of Environmental and Life Sciences, The University of Newcastle, Callaghan NSW 2308, Australia

Full list of author information is available at the end of the article
}

\section{Biomed Central}

(c) 2015 Zhang et al.; licensee BioMed Central. This is an Open Access article distributed under the terms of the Creative Commons Attribution License (http://creativecommons.org/licenses/by/4.0) which permits unrestricted use, distribution, and reproduction in any medium, provided the original work is properly credited. The Creative Commons Public Domain Dedication waiver (http://creativecommons.org/publicdomain/zero/1.0/) applies to the data made available in this article, unless otherwise stated. 


\section{Background}

Transfer cells (TCs) trans-differentiate from a range of existing cell types belonging to the major tissue systems of dermal (e.g., epidermal cells), ground (e.g., endosperm cells; root cortical parenchyma cells) and vascular (phloem and xylem parenchyma cells; companion cells [1]). Once TC development is completed, a 10- to 20fold amplification of their plasma membrane surface area, containing high densities of solute transporters, confer these cells with an extraordinarily high capacity for nutrient exchange between apo- and symplasmic compartments located at bottlenecks for long-distance transport of nutrients throughout the plant body [1]. An intricate invaginated complex of cell wall ingrowths provides structural scaffolding on which the amplified plasma membrane is arrayed to enhance nutrient flows. The tight coupling of this structure/function relationship is graphically illustrated by compromised seed filling of mutants in which TCs, located at the maternal/filial interfaces of both eudicots and monocots, exhibit an attenuated construction of their cell wall ingrowth complex $[2,3]$. Since a number of major crop species, including cereals and grain legumes, contain TCs positioned at the maternal/filial interfaces of their developing seeds [1], the shrivelled seed phenotype exhibited by TC mutants underscores the important role these cells play in determining crop yields.

The cell wall ingrowth complex is organized into one of two architectural types - flange or reticulate [1]. Flange ingrowths form as ribs or bands of wall material while the more commonly occurring reticulate wall ingrowths arise as numerous wall papillae that develop at right angles to the original wall. The extent of their reticulation varies from cylindrical papillae alone to ones in which papillae branch and fuse to generate fenestrated wall layers [4]. Immediately preceding construction of reticulate, but not flange wall ingrowths [5], a structurally distinctive wall-layer, the so-called uniform wall, is rapidly laid down over the pre-existing primary wall of the trans-differentiating cell to a thickness that can extend up to $50 \%$ of that of the pre-existing primary wall [6]. Collectively, the uniform wall and reticulate wall ingrowths form the ingrowth wall [1].

Despite the central role the ingrowth wall, and particularly its wall ingrowth component, plays in underpinning transport function of TCs, little is known about the mechanisms responsible for inducing and then orchestrating its construction. Transcriptional analyses of cells committed to a developmental pathway leading to forming a TC-morphology have identified up-regulated expression of genes encoding components of signalling pathways for abscisic acid, auxin, ethylene, reactive oxygen species (ROS) and $\mathrm{Ca}^{2+}$ in developing seeds [1,7-9] and for auxin and ethylene in giant cells induced by nematode infection [10]. Events downstream from the inductive signals largely have been identified by transcriptome analyses of developing cereal seeds and, in particular, for flange wall ingrowths formed in basal endosperm TCs (BETCs) of maize [11] and barley $[8,12]$. A more limited analysis has been reported for the formation of reticulate wall ingrowths in eudicots $[10,13]$.

While there are undoubtedly some shared features, given the disparate architectures of flange and reticulate wall ingrowths, we hypothesize that signalling pathways, cell wall biosynthesis and delivery of polysaccharides to the wall matrix will have features that are peculiar to each architectural type of ingrowth wall. This characteristic likely extends to distinctive elements responsible for constructing the uniform wall and wall ingrowth papillae. To this end, we used Vicia faba cotyledons, in which on transfer to culture, their adaxial epidermal cells spontaneously undergo trans-differentiation to a TC-morphology [1]. This experimental system provided the opportunity to undertake a transcriptome analysis of TC-specific gene networks [1]. In addition, because of the temporal sequence for the deposition of the uniform wall followed by wall ingrowth papillae, gene cohorts associated with these two developmental events could be distinguished. Hereafter, wall ingrowth papillae, which represent the initial stage of wall ingrowth construction [4], are referred to as wall ingrowths.

\section{Methods}

Plant growth conditions, cotyledon culture and collection of tissue samples for sequencing

$V$. faba L. (cv. Fiord) plants were raised under controlled environmental conditions [13]. Cotyledons of harvested pods were surgically excised and cultured aseptically on liquid MS medium for specified times (see below) before being fixed in $75 \%$ ethanol and $25 \%$ acetic acid for $1 \mathrm{~h}$ at $4^{\circ} \mathrm{C}$. Peels of the adaxial epidermis and blocks of storage parenchyma cells $(2 \times 2 \times 1 \mathrm{~mm})$ were surgically removed from each fixed cotyledon. Collected tissues were immediately snap-frozen in liquid nitrogen and stored at $-80^{\circ} \mathrm{C}$ until used for RNA extraction.

Selection of times to obtain tissue samples from cultured cotyledons was based on the temporal pattern of uniform wall deposition preceding that of wall ingrowths (Figure 1 and, for more information, see Additional file 1). To this end, for the reference library, a representative balance of genes induced to regulate uniform wall and wall ingrowth construction was obtained by collecting $6 \mathrm{mg}$ of epidermal peels from each of freshly harvested cotyledons $(0 \mathrm{~h}$ - reference to identify genes induced/switched off during trans-differentiation) and cotyledons cultured for 1 and $3 \mathrm{~h}$ (dominated by expression of genes regulating uniform wall formation), 6 and $9 \mathrm{~h}$ (dominated by expression of genes regulating deposition of wall ingrowths as uniform wall formation ceased). To identify expression of 


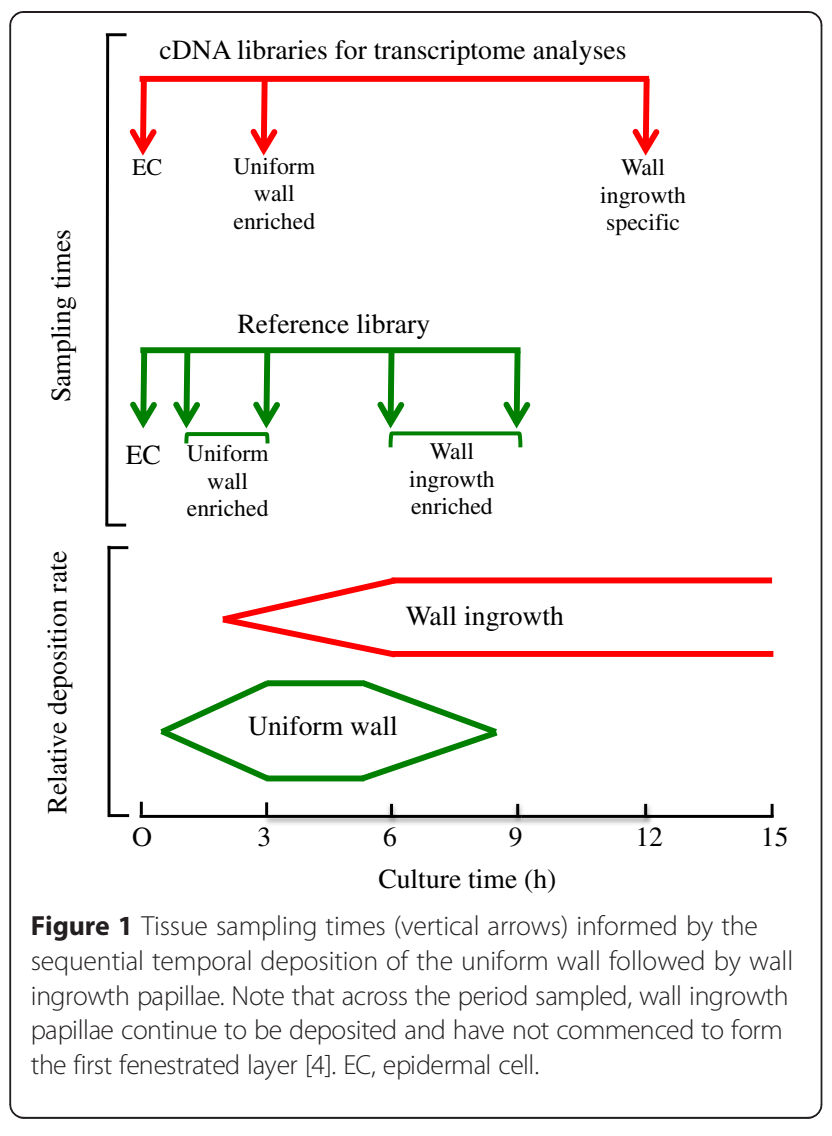

TC-specific genes regulating uniform wall and wall ingrowth construction, epidermal peels $(15 \mathrm{mg})$ and storage parenchyma tissue $(30 \mathrm{mg})$ were sampled from replicate batches of freshly harvested cotyledons and cotyledons cultured for $3 \mathrm{~h}$ (dominated by uniform wall construction) and $12 \mathrm{~h}$ (wall ingrowth construction alone and for additional information see Additional file 1 and Results).

To verify that each sampled population of cultured cotyledons was trans-differentiating, percentages of epidermal cells forming wall ingrowths of a sub-set of cotyledons at $12 \mathrm{~h}$ of culture were scored (see Additional file 1 for Methods).

\section{RNA isolation, cDNA-library construction and Illumina sequencing}

Total RNA was extracted using Qiagen (USA) RNeasy plant mini kits. Contaminating genomic DNA was removed using DNase I.

For preparation of an epidermal-specific TC transcriptome reference library, $1 \mu \mathrm{g}$ of total RNA was aliquoted from the total RNA extracts of each specified harvested time point. The RNA aliquots were combined to generate a temporally mixed RNA library ( $5 \mu \mathrm{g}$ of total RNA). To identify genes responsible for uniform wall and wall ingrowth formation, $2 \mu \mathrm{g}$ aliquots of total RNA were sampled from each biological replicate of epidermal and storage parenchyma RNA extracts.

Total RNA quality was verified by determining the integrity of the 25S and 18S RNA with an Agilent 2100 Bioanalyzer (Agilent, USA and see Additional file 2). The cDNA libraries were prepared from poly-A mRNA isolated from $1 \mu \mathrm{g}$ of total RNA using a TruSeq ${ }^{\circ}$ RNA v2 sample prep kit (Illumina, USA) according to manufacturer's instructions. cDNA quality was evaluated by determining size and purity using an Agilent 2100 bioanalyzer (see Additional file 3). cDNA fragments, ranging from $100-700 \mathrm{bp}$, were selected by agarose gel purification. For the reference library, selected cDNA fragments were $100 \mathrm{bp}$ pair-end sequenced in a single lane on an Illumina HiSeq 2000 platform (Australian Genome Research Facility, Melbourne).

To characterize gene cohorts linked with uniform wall and wall ingrowth deposition, only biological replicates with the anticipated 70\% (or more) of their epidermal cells at $12 \mathrm{~h}$ of cotyledon culture containing detectable wall ingrowths [14] were processed for Illumina sequencing. In three compliant biological replicates for each specified harvest time, cDNA libraries (18 in total) were prepared from total RNA extracts of epidermal peels and storage parenchyma tissues as indicated above (and see Additional files 2 and 3 for RNA and cDNA quality checks). cDNA fragments were purified separately from each sample and indexed with unique nucleic acid identifiers (Illumina TruSeq V2 index sequence). The indexed cDNA libraries were diluted to an average concentration of $10 \mathrm{nM}$ and pooled in equal volumes $(10 \mu \mathrm{L}$ of each library) to generate the final mixed cDNA pool for sequencing. The pool was then $100 \mathrm{bp}$ pair-end sequenced in two lanes on an Illumina HiSeq 2000 platform (Australian Genome Research Facility, Melbourne).

Using Illumina CASAVA pipeline version 1.8.2, raw reads were trimmed with adaptor filtering and a read length cut-off of $50 \%$. Thereafter, filtered reads with over $20 \%$ of their nucleotides having a Q score $<20$ (probability of sequencing error $>0.01$ ) or their sequences having a $\mathrm{N}$ reading over $5 \%$ were removed.

\section{De novo assembly, annotation and GO classification of transcriptome library}

Filtered reads from the cDNA library were assembled de novo into contigs by Beijing Genomics Institution (BGI, Shenzhen, China) using Trinity software release20130225 [15] with a k-mer of 25 and a minimum k-mer threshold abundance of 2 (min_kmer_cov 2). The reads were then mapped back to contigs to assemble unigenes using Trinity Butterfly that filtered out transcriptional artifacts, misassembled transcripts and poorly supported transcripts. Potential redundant sequences were grouped, 
using the TGI Clustering Tool set with a minimum $40 \%$ sequence overlap and over $80 \%$ sequence identity.

Assembled unigene sequences were annotated by alignment to the following publically available databases: NCBI nr (non-redundant, see http://www.ncbi.nlm.nih. gov/refseq/); Swissprot (http://www.uniprot.org/); KEGG (http://www.genome.jp/kegg/); COG (https://www.ncbi. nlm.nih.gov/COG/), using BLASTX with an e-value threshold of $1 \mathrm{e}^{-5}$. Unigenes with no hit in BLASTX were predicted using ESTScan.

\section{Mapping reads to the reference library, determining} differentially expressed genes and GO-enrichment analysis Reads sequenced by the Illumina HiSeq 2000 platform from mRNA extracts of cotyledon epidermal peels and storage parenchyma tissues were analyzed by BGI. Raw reads were filtered as described for the reference transcriptome library. Clean reads were aligned to the reference sequences (generated as described above) using the SOAPallgner/SOAP2 pipeline. No more than 5 mismatches per read were allowed to ensure high quality alignment. Sequence coverage of unigenes in each sample was calculated. During alignment, SOAPallgner/ SOAP2 reported the number of mapped reads per kilo base per million reads (RPKM) as a measure of transcript abundance of each unigene. Transcripts with RPKMs $<0.45$ were considered not to be expressed and were removed from the data sets.

Differentially expressed genes (DEGs) were determined using a computational algorithm based on digital gene expression profiles [16] to perform pairwise differential expression analysis. Multiple testing of the comparisons was corrected using the FDR method [17]. Sequences with a corrected FDR P value of no more than 0.05 were selected. After this correction, genes exhibiting a differential expression of two-fold or more $(\mathrm{P}<0.05)$ were identified as DEGs. Subcellular localization of selected proteins encoded by unigenes was predicted using the WoLF PSORT algorithm.

\section{Quantitative RT-PCR validation}

A collection of 15 unigenes with different expression patterns (see Additional files 4 and 5 for expression pattern information) was selected. cDNA was converted from the same RNA samples sent for Illumina sequencing using QuantiTect Reverse Transcription Kit (Qiagen, USA). Primers were designed using Primer 3 plus (Whitehead Institute for Biomedical Research, USA) and synthesized by Sigma-Aldrich Australia (see Additional file 6 for primer sequences). For each qRT-PCR reaction a $15 \mu \mathrm{L}$ system containing 7.5 $\mu \mathrm{L}$ SYBR Green master mix (Qiagen, USA), $0.375 \mu \mathrm{L}$ of forward and reverse primers $(10 \mu \mathrm{M}), 1.75 \mu \mathrm{L}$ of nuclease free $\mathrm{H}_{2} \mathrm{O}$ and $5 \mu \mathrm{L}$ cDNA was set up. The following PCR cycle was used: $95^{\circ} \mathrm{C}$ for $5 \mathrm{~min}$, $95^{\circ} \mathrm{C}$ for $15 \mathrm{~s}, 60^{\circ} \mathrm{C}$ for $20 \mathrm{~s}, 72^{\circ} \mathrm{C}$ for $30 \mathrm{~s}$; steps 2 to 4 were repeated 50 times. High-resolution melting curves $\left(72-95^{\circ} \mathrm{C}\right)$ following the final PCR cycle checked the specificity of the PCR products. For each cDNA sample, technical duplicates in each of three biological replicates were tested. Four housekeeping gene candidates were assessed using GeNorm. Relative expression levels of each unigene were determined using the two standard curves method.

\section{Results}

De novo assembled transcriptome library for ingrowth wall deposition

Sequencing of reference library cDNA fragments on an Illumina HiSeq 2000 platform generated 181,419,640 pair-end reads (100 bp). After filtering raw reads, $126,200,279$ high-quality reads, in which $97 \%$ of nucleotides have a Phred quality score of $\geq$ Q20 level (error probability $\leq 0.01$ ) (Table 1 ) were de novo assembled into 131,279 contigs (>200 bp) with a N50 value of $980 \mathrm{bp}$ and an average length of $423 \mathrm{bp}$. Clustering yielded 74,659 unigenes with 33,902 consensus sequences grouped into 11,083 distinct clusters and 41,567 singletons (Table 1). This unigene population had a N50 value of $1723 \mathrm{bp}$ and an average length of $1076 \mathrm{bp}$. High integrity and accuracy of the assembly was indicated by $85.2 \%$ of filtered reads mapping to the assembled transcriptome with $41.4 \%$ of reads uniquely mapping to the transcriptome (Table 1 ). The uniquely mapped reads had an average coverage depth of 8.4 (12,620 bp per mRNA).

BLASTX searches of publically available protein databases, using the putative unigenes as query sequences, yielded matches for $68.9 \%$ of the unigenes. BLASTXderived alignments predicted sequence orientation for 43,910 of the unigenes and identified 43,703 open reading frames. Taxonomic distribution of the annotated unigenes by Blast $2 \mathrm{GO}$ demonstrated that over $85 \%$ unigenes had their best hits (smallest e value) with their homologs in other legume species (see Additional file 7). The raw reads and assembled unigene sequences are

Table 1 Raw, filtered and mapped reads used for the de novo assembly of the reference transcriptome library for adaxial epidermal cells of freshly harvested and cultured $V$. faba cotyledons undergoing trans-differentiation to a TC morphology

\begin{tabular}{lcc}
\hline Category & Number & $\begin{array}{c}\text { Percentage (compared with } \\
\text { total number of reads) }\end{array}$ \\
\hline Raw reads & $181,419,640$ & $100 \%$ \\
Clean reads & $126,200,279$ & $69.56 \%$ \\
Mapped reads & $107,093,666$ & $59.03 \%$ \\
Uniquely-mapped reads & $44,339,744$ & $24.44 \%$ \\
Multiple-mapped reads & $62,753,921$ & $34.59 \%$ \\
\hline
\end{tabular}


available at European Nucleotide Archive, accession number: PRJEB8906.

\section{Global gene expression patterns during early phases of trans-differentiation to a TC-morphology}

Total RNA was extracted from epidermal peels and storage tissue blocks at 0,3 and $12 \mathrm{~h}$ of cotyledon culture and processed for RNAseq on an Illumina platform (see Methods). Between 19 - 37 million clean reads were generated for each biological replicate (see Additional file 8 ). An average of $85 \%$ of the clean reads mapped to expressed unigenes. Of these reads, $60 \%$ mapped with a perfect match and $40 \%$ with a mismatch less than 5 bp (Additional file 8). Total numbers of unigenes, detected within epidermal and storage parenchyma cells across cotyledon culture were temporally stable (Additional file 9). Some $70 \%$ of the unigenes exhibited expression levels $>0.45$ RPKM within a cell type and these were dominated by unigenes with expression levels ranging from 0.5 to 10 RPKM (80\%).

An estimated 27,244 unigenes were expressed in adaxial epidermal cells of developing $V$. faba cotyledons (Figure 2A). Of these epidermal cell unigenes, 21,293 were shared with the underlying storage parenchyma cells leaving 5,951 unigenes that were epidermal cell specific (Figure 2A). Upon transiting to a TC fate, expression of 2,805 unigenes in the precursor epidermal cells was switched off (Figure 2A). The combined transcriptomes of trans-differentiating TCs at 3 and $12 \mathrm{~h}$ of cotyledon culture totalling 33,472 unigenes approximated those detected by RNAseq analyses of differentiating BETCs in barley grains $(42,086-[8])$ and of nematode giant TCs in rice roots $(42,756$ unigenes at 7 days post infection; 41,179 genes at 14 days post infection - [18]). Seventy-three \% of the epidermal TC

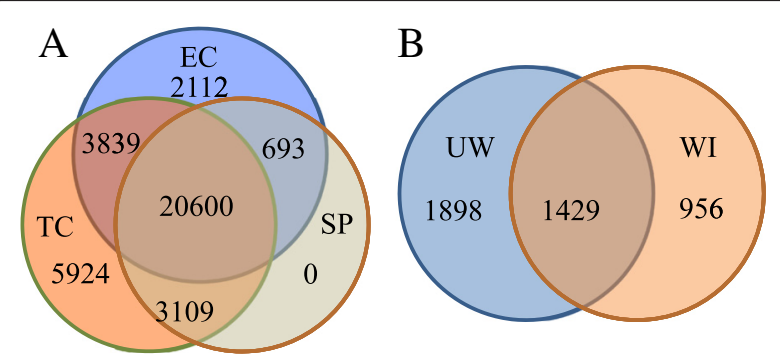

Figure 2 Numbers of unigenes expressed preferentially in, or shared between, (A) cell types and (B) uniform wall and wall ingrowth formation occurring within adaxial epidermal cells of cultured $V$. faba cotyledons. Only unigenes with a RPKM value $>0.45$ in all three biological replicates were defined as 'expressed' in one or more cell types/phases of ingrowth wall deposition. For (B), only unigenes with a coverage of $>70 \%$ were listed for further analysis. EC- adaxial epidermal cell; TC - epidermal TC trans-differentiated from adaxial epidermal cell; SP- storage parenchyma cell; UW - uniform wall; WI- wall ingrowth. transcriptome was represented by ongoing expression of genes by their precursor epidermal cells whilst $27 \%$ were induced (Figure 2A). The percentage of induced genes was comparable to the proportion of upregulated genes in nematode giant TCs [18]. Of the induced genes, $66 \%$ were TC specific and the remainder was shared with the underlying storage parenchyma cells (Figure 2A). Ten \% of the epidermal cell genes were silenced in transiting to an epidermal TC identity and these were replaced 3-fold by induced genes (Figure 2A). TC specific genes were inspected manually to remove duplicates and those with $<70 \%$ coverage to yield a total of 4,283 unigenes (Figure 2B).

\section{Transcriptome networks specifically expressed during uniform wall or wall ingrowth formation}

The temporal sequence of uniform wall followed by wall ingrowth deposition (Figure 1; Additional file 1) provided the opportunity to separate the TC-specific cohort of expressed genes into groups linked with the two phases of ingrowth wall construction and those shared between these phases as follows. The latter were identified by manual inspection of the 3- and 12-h gene expression profiles (Figure 2B). The remaining expressed genes were then specifically linked with either uniform wall or wall ingrowth formation and these were identified on the following grounds. At $12 \mathrm{~h}$ of cotyledon culture, uniform wall construction had ceased and TC-specific gene expression is solely committed to deposition of wall ingrowths (Figure 1; Additional file 1). Thus the expression profile at $12 \mathrm{~h}$ contained transcripts specifically related to constructing wall ingrowths alone (Figure 2B). In contrast, at $3 \mathrm{~h}$ of cotyledon culture, uniform wall construction is proceeding rapidly concurrent with a smaller population of cells starting to deposit wall ingrowths (Figure 1, Additional file 1). Hence at $3 \mathrm{~h}$ of culture, genes involved in uniform wall and wall ingrowth construction would be co-expressed. However, those exclusively directing deposition of wall ingrowths would be expressed at relatively lower levels. Thus subtracting the 12-h (wall ingrowths alone) from the 3-h (uniform wall plus wall ingrowths) gene expression profile identified those genes specifically expressed during uniform wall construction (Figure 2B).

The above exercise demonstrated that of the TCspecific expressed genes, $66 \%$ were selectively linked with uniform wall (44\%) or wall ingrowth (22\%) formation with the remainder (34\%) being expressed throughout ingrowth wall formation (Figure $2 \mathrm{~B}$ and see Additional file 4 for their RPKM gene expression values). This latter cohort of genes was categorised according to their relative expression levels in the two phases of ingrowth wall formation, that is, no change and upregulated during uniform wall or wall ingrowth 
deposition. Genes whose expression levels did not change accounted for $60 \%$ of the common cohort (Table 2).

BlastX searches, at a stringency of $<\mathrm{e}^{-5}$, established that $41 \%$ of the TC-specific genes and $34 \%$ of epidermal cell genes switched off shared homology with known genes listed in public databases (Table 2). The 59/66 \% of un-known transcripts equates with a similar value reported for the transcriptome of developing barley BETCs [12].

Table 2 provides a summary of the changes in expression of selected functional categories of TC-specific genes that are known to be central to TC development and function. For all categories, numbers of epidermal cell genes switched off in transiting to a TC fate were replaced by increased numbers of TC-specific transcripts between 3- to 12-fold. Amongst these, expression of transcription/translation, receptor kinase/kinases, cytoskeleton/vesicle trafficking, matrix polysaccharides and defense genes contributed to the two-fold greater number of genes expressed during uniform wall compared to wall ingrowth construction (Table 2; Figure 2B). In addition, a portion of uniform wall associated expressed genes were linked with a co-occurring burst in cell division/expansion (Additional file 10). Genes encoding the entire flavonol pathway were switched on during TC development (Table 2).

A more detailed analysis was undertaken of the temporal expression of TC-specific genes contained in functional groups that are at the heart of regulating development of the TC's structural specialization of a polarized ingrowth wall that realises their functional capacity to support high rates of nutrient transport across their plasma membranes [1]. These categories included hormonal and $\mathrm{ROS} / \mathrm{Ca}^{2+}$ signal generation and their signalling pathways, cytoskeleton/vesicle trafficking and lipid biosynthetic enzymes related to microdomain formation, cell wall biosynthetic and remodelling enzymes along with cell wall structural proteins and membrane transporters (see Additional files 11, 12, 13 and 14 inclusive). These data are supplemented with estimates of their annotation and relative expression levels at specified stages of ingrowth wall construction along with noting whether their homologues have been detected in transcriptomes of BETCs and nematode giant TCs (Additional file 5). Temporal patterns of expression of selected genes from the targeted functional categories determined by qRT-PCR were consistent with those derived from Illumina sequencing (Additional file 15),

Table 2 Numbers of annotated expressed genes ascribed to specified functional categories for genes switched off in epidermal cells transiting to a TC fate and for those specifically expressed in epidermal cells undergoing trans-differentiation to a TC morphology

\begin{tabular}{|c|c|c|c|c|c|c|}
\hline \multirow[t]{2}{*}{ Functional category } & \multirow{2}{*}{$\begin{array}{l}\text { Epidermal } \\
\text { switched-off } \\
\text { genes }\end{array}$} & \multicolumn{5}{|c|}{ Transfer cell specific expressed genes } \\
\hline & & $\begin{array}{c}\text { UW/WI } \\
\text { no change }\end{array}$ & UW up-regulated & UW specific & WI up-regulated & WI specific \\
\hline Total number of expressed genes & 1381 & 851 & 366 & 1898 & 214 & 958 \\
\hline Total number of annotated genes & 468 & 364 & 217 & 647 & 138 & 407 \\
\hline DNA synthesis and modelling & 17 & 8 & 1 & 13 & 2 & 13 \\
\hline Transcription/translation & 32 & 27 & 11 & 31 & 3 & 19 \\
\hline \multicolumn{7}{|l|}{ Signalling } \\
\hline Receptor kinase/kinase & 15 & 17 & 20 & 37 & 8 & 16 \\
\hline Hormonal & 4 & 6 & 6 & 13 & 8 & 14 \\
\hline $\mathrm{ROS} / \mathrm{Ca}^{2+}$ & 1 & 4 & 9 & 6 & 1 & 6 \\
\hline \multicolumn{7}{|l|}{ Intracellular organization } \\
\hline Cytoskeleton/vesicle trafficking & 10 & 6 & 2 & 11 & 1 & 7 \\
\hline Membrane microdomains & 0 & 3 & 5 & 3 & 1 & 7 \\
\hline \multicolumn{7}{|l|}{ Cell wall enzymes and structural proteins } \\
\hline Cellulose & 3 & 0 & 1 & 3 & 1 & 1 \\
\hline Matrix polysaccharides & 9 & 4 & 6 & 14 & 3 & 7 \\
\hline Structural proteins & 2 & 3 & 3 & 3 & 5 & 5 \\
\hline Membrane transporters & 14 & 13 & 9 & 12 & 1 & 13 \\
\hline Defense & 4 & 10 & 21 & 12 & 1 & 6 \\
\hline Flavonoid synthesis and compartmentation & 5 & 6 & 4 & 3 & 4 & 8 \\
\hline
\end{tabular}

TC specific genes separated into those genes expressed throughout uniform wall (UW) and wall ingrowth (WI) formation and those that are specific to each of these wall-building phases. Genes expressed throughout ingrowth wall formation are separated into groups depending on their differential expression patterns of no change, up-regulated during UW or WI formation (for more details, see Results). 
suggesting that the Illumina data are relatively reliable (Table 2, Additional files 4, 5, 11, 12, 13, and 14).

\section{Discussion}

The ability to obtain peels of cotyledon epidermal cells permitted cell-specific transcriptomes of these cells to be identified as they trans-differentiated to a TC-morphology [13]. In addition, developmental stage-specific transcriptomes were isolated (Figure 2, Additional files 5, 11, 12, 13 , and 14) by taking advantage of the temporal disjunction between uniform wall and wall ingrowth construction (Figure 1, Additional file 1). We interrogated these TCspecific transcriptomes to discover genes encoding proteins of functional categories considered to contribute to developing a transport capable TC (Figure 3 and see Additional files 5, 11, 12, 13, and 14). Transcriptional cascades are initiated by auxin and ethylene $[13,19,20]$ along with $\mathrm{ROS}$ and $\mathrm{Ca}^{2+}$ serving as secondary messengers and positional signals $[6,9]$. The substantial numbers of expressed receptor kinases/kinases and transcription factors, point to these forming a significant component of the signalling network (Table 2 and see [12]). Re-organization of the cytoskeleton and vesicle trafficking together with membrane microdomains, under control of the inductive signalling

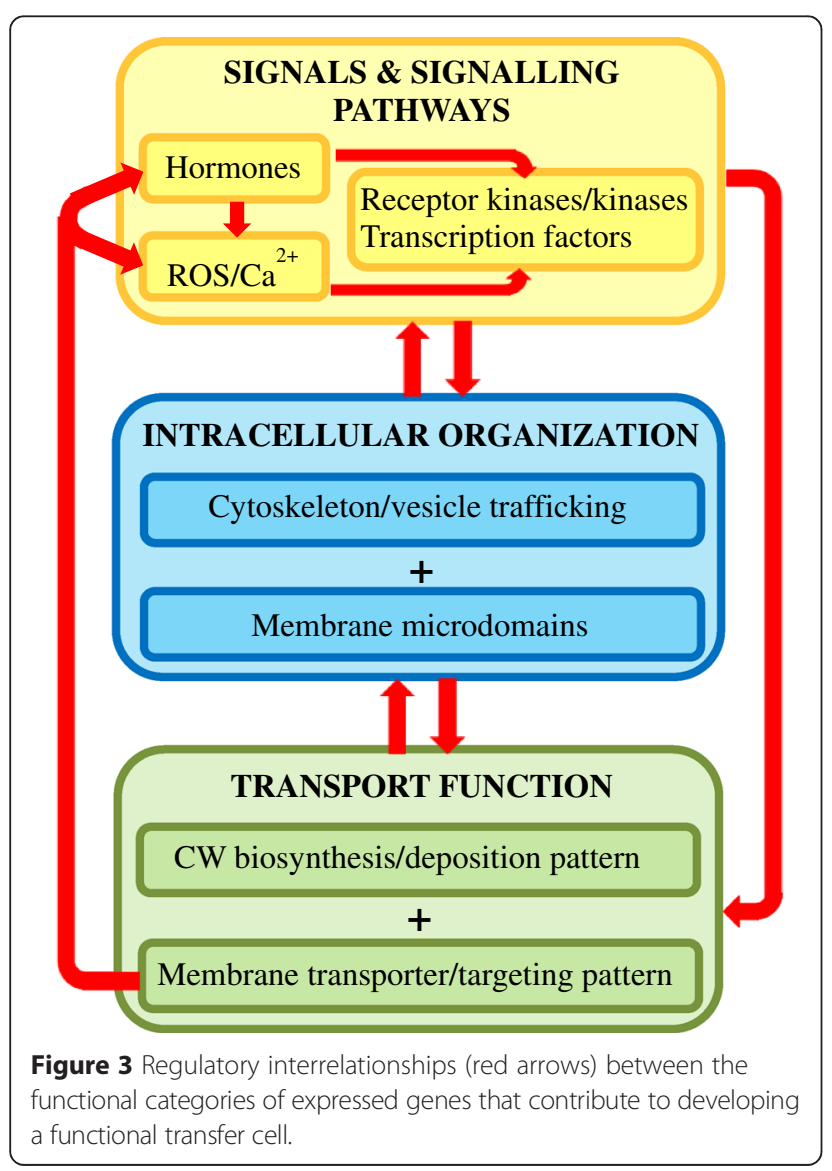

network, would be expected to direct targeted delivery of cargos containing cell wall biosynthetic enzymes and cell wall components to construct the polarized uniform wall and localized wall ingrowths (Figure 3 and Additional file 1). The latter provide a scaffold to support an amplified plasma membrane surface area to which membrane transporters are targeted through a polarized endomembrane secretory system to confer enhanced TC transport function (Figure 3). In addition, targeted positioning of transporters is responsible for generating the polarized extracellular ROS [6] and cytosolic $\mathrm{Ca}^{2+}$ [9] signals and possibly altering positioning of auxin transporters that may contribute to generating the auxin maximum ([13]; Figure 3). The following discussion focuses on expressed genes belonging to these three functional categories namely, signals and signalling pathways, intracellular organization and transport function (Figure 3). The discussion starts with seeking out candidates responsible for generating the developmental signals and downstream signalling pathways. In all cases, statements regarding gene identity and function should be considered as putative.

\section{Signals and signalling pathways}

Hormonal signals, acting in series with $\mathrm{ROS}$ and $\mathrm{Ca}^{2+}$ [1], likely activate expression of receptor kinases/kinases and transcription factors to induce TC trans-differentiation (Figure 3).

\section{Auxin, ethylene and abscisic acid form a complex of primary signals putatively regulating $T C$ development} Consistent with previous findings for epidermal TCs $[13,19]$ and those reported for nematode giant TCs (10), induction of trans-differentiation was accompanied by changes in auxin activity (as determined by expression of auxin-induced genes) increasing five-fold between uniform wall and wall ingrowth formation driven by an auxin maxima generated by enhanced auxin biosynthesis and disrupted polar transport (Additional file 11). For instance, auxin biosynthesis could be elevated by expression of indole-3-acetate O-methyltransferase IAA, that catalyzes synthesis of a biologically more active IAA, methyl-indole-3-acetic acid [21] and indole-3-glycerol phosphate synthase that starts the tryptophan independent IAA biosynthetic pathway [22]. Polar auxin transport was likely disrupted by de-localized distribution of expressed auxin efflux and influx carriers by coexpression of KAN and HD-ZIPIII [23]. As a point of distinction between mechanisms regulating flange and reticulate wall ingrowths, in contrast to the latter $[10,13,19]$, differentially expressed auxin-related genes were not detected in developing BETCs $[8,11]$.

Initiation of epidermal TC development is linked with a substantive burst in ethylene biosynthesis, regulated by 
an auxin-induced expression of ACC synthases [19], driving expression of ethylene response factors (ERFs) [19] and particularly so for uniform wall formation (Additional file 11). For example, ERF2.6 and SHINE expression could invoke cell wall biosynthesis through respectively up-regulating callose and cellulose biosynthesis (Additional file 13 and see [24,25]). Strong expression of ethylene signalling genes during uniform wall formation corresponded with that reported for early phases of wall ingrowth construction in barley BETCs [8] and nematode giant TCs [10].

A switch to a specific ABA signalling pathway throughout ingrowth wall formation was suggested by expression of PYL6, an ABA receptor [26], $L E C 1$, tightly connected with $A B A$ signalling regulating $A B 13$ and $A B 15$ [27] and ERF RAP2.6 that acts through an AB14-mediated signalling pathway ([28]; Additional file 11). ABA also acts during wall ingrowth formation (Additional file 11). ABA action throughout ingrowth wall formation in epidermal TCs contrasts with that for barley BETCs where ABA induces a maturation program once wall ingrowth deposition ceases [8].

Three gibberellin biosynthetic genes with the biological activities of their products being modulated by two gibberellin 2-oxidases and Short Internode Related during uniform wall formation are expressed (Additional file 11). These gibberellins likely regulate cell division and expansion (Additional file 10 and see [29]). An absence of gibberellin activity during wall ingrowth construction was suggested by co-expression of Short Internode Related and gibberellin 2-beta-dioxygenase 8like (Additional file 11; [29]). Residual cytokinin levels in the developing epidermal TCs would be depleted through degradation by the expressed cytokinin dehydrogenase [30] and by conjugation to an inactive form catalyzed by the five cytokinin-O-glucosyltransferases ([31]; Additional file 11). No components of the salicylic acid or brassinosteroid signalling pathways were detected in the TC-specific transcriptome (Additional files 4 and 11) indicating that these pathways likely did not play a role in trans-differentiation to a TC state.

\section{Reactive oxygen species and $\mathrm{Ca}^{2+}$ serve as secondary messengers directing ingrowth wall construction}

Extracellular ROS, produced by ethylene-induced respiratory burst oxidases (rbohs), regulate biosynthesis and polarized deposition of the uniform wall in epidermal TCs [6,32]. Similarly, rboh expression co-occurs with wall ingrowth formation in barley BETCs [8]. In contrast, no differentially expressed rbohs were detected in nematode giant TCs [10]. Interestingly, ROS-related gene expression was dominated by those involved in extra- and intracellular ROS homeostasis (Additional file 11) suggesting that ingrowth wall formation depends upon a spatio/temporal fine-tuning of ROS. For instance, antioxidant capacity could be down regulated by ascorbic acid oxidation catalyzed by an extracellular L-ascorbate oxidase ([33]; Additional file 11) whilst concurrent ROS degradation by peroxidation [32] could be mediated by carboxymethylenebutenolidase-like protein (Additional file 11), secreted to the cell wall [34]. In contrast to epidermal TCs, differentially expressed peroxidases were not detected in barley BETCs [8] and were strongly downregulated in nematode giant TCs [10].

Loci at which wall ingrowths are deposited are defined by a cytosolic $\mathrm{Ca}^{2+}$ signal spatially organized into narrow plumes shaped by co-operative activities of plasma membrane $\mathrm{Ca}^{2+}$-permeable channels organized into clusters surrounded by $\mathrm{Ca}^{2+}$-ATPases [9]. However, unlike the unknown DHP receptor $\mathrm{Ca}^{2+}$-permeable channels responsible for generating the $\mathrm{Ca}^{2+}$ signal [9], ion conductances of the expressed SKOR, annexin and cyclic nucleotide gated channels are not inhibited by verapamil or nifedipine (e.g. [35]). This, together with channel expression coinciding with uniform wall construction (Additional file 11), points to an additional role for $\mathrm{Ca}^{2+}$ signalling in transiting precursor epidermal cells to a TC fate. A conclusion that accommodates $\mathrm{Ca}^{2+}$ signalling genes being expressed in both flange [8] and reticulate forms $[18,36]$ of developing TCs. $\mathrm{Ca}^{2+}$ signalling would appear to be mediated through $\mathrm{Ca}^{2+}$-dependent protein kinases during uniform wall formation while a calmodulin signalling route could operate when wall ingrowths are being deposited as suggested by expression of three IQ-domain containing proteins (Additional file 11) that bind calmodulins to facilitate their function.

\section{Receptor kinases/kinases represent a significant component} of the signalling network

TC-specific receptor kinases/kinases represented a major component of the signalling networks (59\%) expressed within the epidermal TCs (Table 2). While their functions are unknown, similar to nematode giant TCs [10], members of a two component signalling system present in barley BETCs [12], was not detected in the epidermal TC cohort of receptor kinases/kinases. Thus, the presence/absence of a two component signalling system could underpin the distinction between flange [12] and reticulate ([10]; this study) TC wall architectures.

\section{Intracellular organization}

Polarized accumulation of extracellular ROS regulating uniform wall synthesis [6] and cytosolic $\mathrm{Ca}^{2+}$-plumes determining loci for wall ingrowth formation [9] respectively depend upon a finely-tuned intracellular distribution of rbohs and $\mathrm{Ca}^{2+}$-permeable channels. This requirement is undoubtedly met through modifying cytoskeleton and vesicle trafficking combined with re-configuring microdomains in 
the plasma membrane abutting sites of ingrowth wall deposition (Figure 3).

\section{TC-specific expression of cytoskeleton and vesicle trafficking confers TC polarity}

Microtubule and actin-associated genes switched off on transiting to a TC fate were predominantly linked to cell division (Additional file 12) that declined rapidly from three to six hours (Additional file 10). Consistent with polarized cell wall deposition [4], TC- specific genes indicative of remodelling cytoskeletal elements [37] were expressed either across ingrowth wall deposition (kinesin motor domain and formin-like protein) or only during one phase of the process (Additional file 12). Uniform wall specific gene expression (Additional file 12) indicated substantial polymerization/reorganization of actin filaments (actin and actin-97-like) and polarization of transport vesicles and organelles (Myosin X1) [38] consistent with ROS-regulated polarization of the uniform wall [6]. Wall ingrowth specific expression of $65-k D a$ microtubule-associated protein 3-like and Villin-4 respectively suggests establishing a stable microtubule [39] and actin [40] network to orchestrate deposition of wall ingrowths at loci. In root hair growth of Arabidopsis, AtVLN4 regulated actin organization is $\mathrm{Ca}^{2+}$-dependent [41] pointing to an interaction between actin and $\mathrm{Ca}^{2+}$ plumes that define wall ingrowth loci [9].

Rapid polarized/localized cell wall deposition during formation of the ingrowth wall (Additional file 1) is dependent upon exo/endocytotic activity [13]. Regulation of cycling of ER-derived proteins through assembly and directed transport of coat protein complex II (GTPbinding protein SAR1A and ER-derived vesicle protein ERV14) and ARFs (ADP-ribosylation factor GTPaseactivating proteins) across ingrowth wall deposition is consistent with asymmetrical vesicle trafficking to effect polarity [42,43]. Expression of uniform wall- and wall ingrowth-specific exocyst complex components and $\mathrm{v}$ - and t-SNARES (Vesicle-associated protein 2-1-like, Vesicleassociated membrane protein and two, Syntaxin -112 genes), to direct targeted vesicle delivery to the plasma membrane [44], confirms the specialized nature of each phase of ingrowth wall deposition. Two of four genes encoding proteins involved in membrane trafficking (Dynamin -related protein 1C-like and Dynamin-2Blike) are potential regulators of localized endocytosis supporting wall ingrowth deposition. These two dynamin genes represent two subfamilies of DRPs that interact and assemble with clathrin at discrete foci in the plasma membrane of Arabidopsis cells to regulate endocytosis [45] and cellulose deposition [46]. This profile of cytoskeleton and vesicle trafficking genes is comparable to that reported for BETCs [8] and nematode giant TCs [18].

\section{Formation of membrane microdomains may sub-} compartmentalize the plasma membrane polar domain

Sphingolipids and sterols form major components of membrane microdomains [47]. Indeed, genes encoding enzymes in their biosynthetic pathways are expressed during development of flange wall ingrowths in barley BETCs [8]. Sphingolipids consist of a polar head group linked to an amino alcohol long-chain base (LCB) with the amine group acylated with a fatty acid (ceramide) with C4 of LCB being (trihydroxy) or not being (dihydoxy) hydroxylated. Bax inhibitor 1-like (Additional file 12) post-translationally activates the $\mathrm{C} 4$ hydrolase [48] thus promoting flux through this route for acylation by very long-chain fatty acids (VLCFA; [49]). Significantly, synthesis of VLCFAs may be enhanced by expression of four 3-ketoacyl-CoA synthase isoforms, a elongation of fatty acid protein and a longchain-fatty-acid-CoA ligase (Additional file 12) that form part of the elongase complex [50]. An increased precursor flow into the VLCFA pathway is supported by up-regulated expression of biotin carboxyl carrier protein of acetyl-CoA carboxylase (Additional file 12), a sub-component of acetyl-CoA carboxylase catalyzing the first carboxylation step in, and rate limiting of, de novo fatty acid synthesis [51]. Thereafter, bax inhibitor 1-like may also increase activities of fatty acid hydroxylases and desaturases that further modify the ceramides [48] before synthesis of the final sphingolipid products of glucosylceramides or glycosyl inositolphosphoceramides [49]. Uniform wall specific expression of an oxysterol-binding protein-related protein and a remorin (Additional file 12) respectively point to vesicle trafficking of sterols [52] and their enrichment in membrane microdomains [47]. Wall ingrowth deposition is linked with expression of putative sterol biosynthetic genes (Additional file 12) such as two epoxide hydrolases [53] and squalene epoxidase [54].

\section{TC transport function}

Expression of cell wall synthesizing and re-modelling enzymes underpins construction of the ingrowth wall that supports the amplified plasma membrane in which are embedded high densities of membrane transporters to collectively confer an enhanced transport function (Figure 3).

\section{Expression of cell wall biosynthesis and re-modelling genes construct the ingrowth wall}

Cellulose is present in the uniform wall and the inner, electron dense region, of wall ingrowths of epidermal TCs [55] but cellulose biosynthesis appeared not to depend on expression of TC-specific CesAs that were switched off and not replaced as the epidermal cells transited to a TC fate (Additional file 13). Rather, cellulose deposition could be regulated post-translationally 
(Additional file 13). Thus during uniform wall formation, SHINE [56] and a GPI-anchored protein [57] could regulate CesA activity with the extruded cellulose microfibrils being re-modelled by two extracellular endoglucanases [58]. Cellulose provides a structural scaffold to form wall ingrowths at right angles to the uniform wall [59]. Here, an acid endochitinase-like protein, known to associate with cellulose synthase complexes [25] and an extracellular beta-glucosidase, may form part of the machinery redirecting extrusion of cellulose microfibrils (Additional file 13). Consistent with these observations, CesA expression is down-regulated in nematode giant TCs [36] while in maize and barley BETCs, CesA expression is upregulated during later stages of TC development $[8,11]$.

In contrast to cellulose biosynthesis, there was a substantial TC-specific induction of genes encoding enzymes responsible for synthesizing and re-modelling cell wall matrix polysaccharides (Table 2) and, in particular, hemicelluloses (Additional file 13). To this end, a (1-3,1-4) $\beta$-D-glucan synthase, was switched off and replaced with enzymes catalyzing xylan biosynthesis (xylosyltransferases, glycotransferase family GT8 protein - [60]) and remodelling (beta-D-xylosidase - [61]). Xylans in the uniform wall are consistent with their presence in flange wall ingrowths as suggested by expression of a beta- $D$ xylosidase and a xylosyltransferase respectively in BETCs of barley [8] and maize [11]. Xyloglucan presence in uniform walls [55] is consistent with expression of xylosyltransferases (biosynthesis - [62]) and endoxylotransglucosylase/hydrolases (chain re-modelling [63]). Xyloglucan biosynthesis and re-modelling also dominates early developmental stages of barley BETCs [8] and nematode giant TCs [10]. Xyloglucan re-modelling coincides with TC division/expansion as indicated by coexpression of expansins ([8,10,11]; Additional files 10 and 13 in this study). In contrast, expansins expressed during wall ingrowth construction when cell division/expansion has ceased (Additional files 10 and 13), must serve a yet-to-be identified function. One possibility is to free xyloglucan chains from cellulose microfibrils rendering them available for co-operative re-modelling by alphaxylosidases and a beta-glucosidase [64] and by two extracellular isoforms of beta-galactosidase ([58] Additional file 13). Overall, uniform wall and wall ingrowth construction was characterized by intense xyloglucan remodelling.

Pathways leading to pectin biosynthesis and modification altered on epidermal cells transiting to a TC fate (Additional file 13). During uniform wall construction, synthesis of the pectin rhamnogalacturon backbone (RG1) was likely catalyzed by expression of galactofuronosyl-transferase 13-like (GAUT13 - Additional file 13) that significantly is essential for polarized pollen tube tip growth [65]. Control over the pectin gel state is conferred by co-expression of a pectin esterase inhibitor modulating catalytic activities of expressed pectin esterases (Additional file 13) that catalyze removal of methyl ester groups from homogalacturonan backbones to allow their cross-linking with $\mathrm{Ca}^{2+}[66]$. This accounts for esterified pectins being the predominate type present in the ingrowth wall [55]. Up-regulated and wall ingrowth specific expression of two beta-galactosidases could catalyze cleavage of terminal galactosyl residues from RGI [67]. Overall, uniform wall formation included pectin biosynthesis and re-modelling giving way to pectin re-modelling alone for wall ingrowth deposition. Enhanced pectin biosynthesis and re-modelling also characterizes construction of barley BETCs [8] and nematode giant TCs [36].

Callose deposition is a balance between synthesis by callose synthases and degradation by $\beta-1,3$-glucanases. Isoforms of both enzymes were switched off in epidermal cells on transiting to a TC fate and were not replaced during uniform wall formation (Additional file 13). However, UDP-glucuronosyltransferase 1 (UGT1), a callose synthase complex component essential for callose synthesis [68], was expressed during wall ingrowth construction accompanied by two beta-1, 3-glucanase isoforms (Additional file 13); an expression pattern consistent with callose being confined to the outer sheath enveloping each wall ingrowth [55]. This temporal profile of callose deposition corresponds with that reported for nematode giant TCs [36] but contrasts with callose synthesis/turnover being restricted to the cell division phase of barley BETC development [69].

Transcripts encoding enzymes of the phenylpropanoid pathway occurred across ingrowth wall formation (Additional file 13). Expressed ABC transporters (Additional file 14) could efflux the monolignol precursors to the cell wall where class III peroxidases (Additional file 11) catalyze their polymerization to lignin [70]. Significantly, the full complement of phenylpropanoid pathway enzymes is expressed in nematode giant TCs [36], and a detailed histochemical study of maize BETCs [71], provides strong evidence for their lignification.

Extensins are expressed in TCs forming flange $[8,11]$ and reticulate [18] wall ingrowths. A large number (14) of extensin family members of hydroxyproline-rich glycoproteins were expressed in developing epidermal TCs (Additional file 13), implying a key role played by extensins in shaping the ingrowth wall and, in particular, wall ingrowths. A key enzyme in the post-translational modification pathway generating functional extensins, prolyl 4-hydroxylase [72], was expressed throughout ingrowth wall formation (Additional file 13). Upon being exocytosed into the cell wall space, extensin monomers are envisaged to cross-link to form networks, mediated by 
redox activity of type-III apoplasmic peroxidases ([72]; Additional file 11). Expression of a proline-richextensin-like receptor kinase (Additional file 3), regulating extensin/pectin interactions [73], may generate supramolecular structures [72] to function as templates for ingrowth wall formation. Significantly, coincident with wall ingrowth construction, was the expression of two nodule-specific extensins that support polarized tip growth in elongating infection threads [74] and a DZHRGP homolog that contributes to polarized tip growth of pollen tubes [72].

\section{A large array of membrane transporters is specifically expressed in $T C S$}

The large number of transporter genes switched off upon transiting to an epidermal TC fate (Additional file 14) suggests a substantial change in transport function.

A striking feature of the 13 TC-specific membrane transporters, whose expression did not change during trans-differentiation, nine transport inorganic and organic nitrogen compounds (Additional file 14). Five transport amino nitrogen compounds including two aquaglyceroporins (NIP1-2; nodulin26 - [75]; Additional file 14). Two nucleoside proton symporters, adenine/ guanine permease AZG1 [76] and equilibrative nucleoside transporter 3 [77], likely function to retrieve apoplasmic nucleosides released from degradation of endosperm and inner cell layers of seed coats crushed by expanding cotyledons. Collectively, expression of these transporters reflects a demand for nitrogenous precursors to support protein biosynthesis. Expression of a putative plasma membrane potassium transporter and tonoplast potassium proton antiporter (Additional file 14) ensure potassium homeostasis.

Investment into nitrogen transporters was enhanced during uniform wall formation (Additional file 14). This included a chloride channel (CLC-7 - Additional file 14) that may function as a nitrate sensor to regulate development by modulating auxin transport [89]. Two additional transporters were recruited for potassium homeostasis; a SKOR channel (Additional file 11; [35]) and a potassium antiporter (Additional file 14). Most pronounced was the large number (12) of expressed $A B C$ transporter genes (Additional file 14) linked with expression of flavonoid biosynthetic and defense genes (Table 2). However, along with effluxing xenobiotics, members of the $\mathrm{ABCB}$ transporter subfamily facilitate plasma membrane transport of IAA [78] contributing to the altered IAA homeostasis of epidermal TCs (see Additional file 11 and associated text).

With the onset of wall ingrowth deposition, appearance of two sugar transporter transcripts (Additional file 14) reflects a growing dependence on an extracellular carbon supply [79]. Further investments were made in amino nitrogen and potassium transporters (Additional file 14). An ongoing expression of $\mathrm{ABC}$ transporters (Additional file 14) corresponded with expression of flavonoid biosynthesis and defense genes (Table 2).

\section{Conclusions}

In epidermal cells undergoing trans-differentiation to a TC-morphology, only a small proportion of their transcriptome (i.e., 10\%) was found to be TC-specific. Within the cohort of TC-specific transcripts, those encoding pathways regulating signalling and intracellular organization were fine-tuned whilst cell wall construction and membrane transporter profiles were altered substantially upon transiting to a TC fate. Each phase of ingrowth wall construction was linked with an unique sub-cohort of expressed genes. Comparison of transcriptomes of cells forming flange or reticulate wall ingrowths detected subtle differences in their transcript profiles, with the major difference being the absence of a twocomponent signalling system from the latter.

\section{Availability of supporting data}

The cDNA sequence datasets of raw reads and assembled reference transcriptome library supporting the results of this article are available in the repository of the European Nucleotide Archive (ENA) with the ENA accession number: PRJEB8906.

\section{Additional files}

\section{Additional file 1: Figure S1, Table S1 and accompanying text. \\ Temporal formation of the uniform wall and wall ingrowths.}

Additional file 2: Figure S2. Quality assessment of total RNA extracted from adaxial epidermal and storage parenchyma cells of $V$. faba cotyledons.

Additional file 3: Table S2. Quality assessment of total RNA extracted from, and cDNA libraries of $V$. faba cotyledons used for Illumina sequencing.

Additional file 4: Table S3. Annotation and expression levels of all genes specifically expressed in the epidermal cells of cultured $V$. faba cotyledons trans-differentiating to epidermal transfer cells.

Additional file 5: Table S4. Annotation and expression levels (RPKM) of selected unigenes expressed specifically in trans-differentiating adaxial epidermal cells of $V$. faba cotyledons [80].

Additional file 6: Table S5. Primer sequences used for qRT-PCR determination of expression levels of specified genes to validate RNAseq data sets.

Additional file 7: Figure S3. Species distribution of genes with highest sequence similarity to unigenes in the reference transcriptome library of trans-differentiating adaxial epidermal cells of cultured $V$. faba cotyledons.

Additional file 8: Table S6. Reads generated from Illumina sequencing used to identify gene cohorts linked with uniform wall and wall ingrowth deposition in trans-differentiating epidermal cells of $V$. faba cotyledons.

Additional file 9: Table S7. Temporal profile of unigene numbers expressed in adaxial epidermal or storage parenchyma cells of cultured V. faba cotyledons.

Additional file 10: Figure S4 and associated text. Cell division and expansion of adaxial epidermal cells of cultured $V$. faba cotyledons.

Additional file 11: Table S8. Genes encoding proteins generating or transducing developmental signals switched off in epidermal cells 
transiting to a TC fate and those specifically expressed in epidermal cells undergoing trans-differentiation to a TC morphology.

Additional file 12: Table S9. Genes encoding proteins involved in regulation of the cytoskeleton and vesicle trafficking and sphingolipid/ sterol synthesis and transport that are switched off in epidermal cells transiting to a TC fate and those specifically expressed in epidermal cells undergoing trans-differentiation to a TC morphology.

Additional file 13: Table S10. Genes encoding cell wall biosynthetic and remodelling enzymes and structural proteins switched off in epidermal cells transiting to a TC fate and those specifically expressed in epidermal cells undergoing trans-differentiation to a TC morphology.

Additional file 14: Table S11. Genes encoding membrane transporters switched off in epidermal cells transiting to a TC fate and those specifically expressed in epidermal cells undergoing trans-differentiation to a TC morphology.

Additional file 15: Figure S5. Validation of expression patterns of unigenes obtained from lllumina sequencing using quantitative RT-PCR.

\section{Competing interests}

The authors declare that they have no competing interests.

\section{Authors' contributions}

JWP and CEO initiated the research and designed the experimental approach. $H M Z, X X$ and SW performed the experiments. HMZ processed the data and HMZ, JWP, CEO, RR and HW interpreted the results and prepared the manuscript. All authors have read and approved the final manuscript.

\section{Acknowledgements}

The research was supported by funding from the Australian Research Council (DP130101396) awarded to CEO and JWP together with funding from the German Research Foundation (DFG; grant GZ RA 2061/3-1) awarded to RR. XX and SW are grateful for the support of Faculty Research Higher Degree scholarships. We are indebted to Joseph Enwright for a continuous supply of healthy experimental plant material. The SEM and TEM observations were completed in The University of Newcastle EMX-ray Unit.

\section{Author details}

'School of Environmental and Life Sciences, The University of Newcastle, Callaghan NSW 2308, Australia. '2eibniz Institute of Plant Genetics and Crop Plant Research (IPK), Corrensstrasse 3, D-06466, Gatersleben, Germany.

\section{Received: 9 February 2015 Accepted: 1 April 2015}

\section{Published online: 16 April 2015}

\section{References}

1. Andriunas FA, Zhang HM, Xia X, Patrick JW, Offler CE. Intersection of transfer cells with phloem biology-broad evolutionary trends, function, and induction. Front Plant Sci. 2013;4:221.

2. Borisjuk L, Wang TL, Rolletschek H, Wobus U, Weber H. A pea seed mutant affected in the differentiation of the embryonic epidermis is impaired in embryo growth and seed maturation. Development. 2002;129(7):1595-607.

3. Costa LM, Yuan J, Rouster J, Paul W, Dickinson H, Gutierrez-Marcos JF. Maternal control of nutrient allocation in plant seeds by genomic imprinting. Curr Biol. 2012;22(2):160-5.

4. Talbot MJ, Franceschi VR, McCurdy DW, Offler CE. Wall ingrowth architecture in epidermal transfer cells of Vicia faba cotyledons. Protoplasma. 2001;215(1-4):191-203.

5. Monjardino P, Rocha S, Tavares AC, Fernandes R, Sampaio P, Salema R, et al. Development of flange and reticulate wall ingrowths in maize (Zea mays L.) endosperm transfer cells. Protoplasma. 2013;250(2):495-503.

6. Andriunas FA, Zhang HM, Xia X, Offler CE, McCurdy DW, Patrick JW. Reactive oxygen species form part of a regulatory pathway initiating transdifferentiation of epidermal transfer cells in Vicia faba cotyledons. J Exp Bot. 2012;63(10):3617-29.

7. Drea S, Leader DJ, Arnold BC, Shaw P, Dolan L, Doonan JH. Systematic spatial analysis of gene expression during wheat caryopsis development Plant Cell. 2005;17(8):2172-85.
8. Thiel J, Riewe D, Rutten T, Melzer M, Friedel S, Bollenbeck F, et al. Differentiation of endosperm transfer cells of barley: a comprehensive analysis at the micro-scale. Plant J. 2012;71(4):639-55.

9. Zhang HM, Imtiaz MS, Laver DR, McCurdy DW, Offler CE, van Helden DF, et al. Polarized and persistent $\mathrm{Ca}^{2+}$ plumes define loci for formation of wall ingrowth papillae in transfer cells. J Exp Bot. 2015;66:1179-90.

10. Cabrera J, Barcala M, Fenoll C, Escobar C. Transcriptomic signatures of transfer cells in early developing nematode feeding cells of Arabidopsis focused on auxin and ethylene signaling. Front Plant Sci. 2014;5:107.

11. Xiong Y, Li Q-B, Kang B-H, Chourey P. Discovery of genes expressed in basal endosperm transfer cells in maize using 454 transcriptome sequencing. Plant Mol Biol Rep. 2011;29(4):835-47.

12. Thiel J, Hollmann J, Rutten T, Weber H, Scholz U, Weschke W. 454 Transcriptome sequencing suggests a role for two-component signalling in cellularization and differentiation of barley endosperm transfer cells. PLoS One. 2012;7(7), e41867.

13. Dibley SJ, Zhou Y, Andriunas FA, Talbot MJ, Offler CE, Patrick JW, et al. Early gene expression programs accompanying trans-differentiation of epidermal cells of Vicia faba cotyledons into transfer cells. New Phytol. 2009;182(4):863-77.

14. Wardini T, Wang XD, Offler CE, Patrick JW. Induction of wall ingrowths of transfer cells occurs rapidly and depends upon gene expression in cotyledons of developing Vicia faba seeds. Protoplasma. 2007;231(1-2):15-23.

15. Grabherr MG, Haas BJ, Yassour M, Levin JZ, Thompson DA, Amit I, et al. Full-length transcriptome assembly from RNA-Seq data without a reference genome. Nat Biotechnol. 2011;29(7):644-52.

16. Audic S, Claverie JM. The significance of digital gene expression profiles. Genome Res. 1997;7(10):986-95.

17. Benjamini Y, Drai D, Elmer G, Kafkafi N, Golani I. Controlling the false discovery rate in behavior genetics research. Behav Brain Res. 2001;125(1-2):279-84.

18. Ji H, Gheysen G, Denil S, Lindsey K, Topping JF, Nahar K, et al. Transcriptional analysis through RNA sequencing of giant cells induced by Meloidogyne graminicola in rice roots. J Exp Bot. 2013;64(12):3885-98.

19. Zhou Y, Andriunas F, Offler CE, McCurdy DW, Patrick JW. An epidermal-specific ethylene signal cascade regulates trans-differentiation of transfer cells in Vicia faba cotyledons. New Phytol. 2010;185(4):931-43.

20. Andriunas FA, Zhang HM, Weber H, McCurdy DW, Offler CE, Patrick JW. Glucose and ethylene signalling pathways converge to regulate trans-differentiation of epidermal transfer cells in Vicia narbonensis cotyledons. Plant J. 2011;68:987-98.

21. Zhao N, Guan J, Lin H, Chen F. Molecular cloning and biochemical characterization of indole-3-acetic acid methyltransferase from poplar. Phytochemistry. 2007;68(11):1537-44.

22. Mano Y, Nemoto K. The pathway of auxin biosynthesis in plants. J Exp Bot. 2012;63(8):2853-72.

23. Huang T, Harrar Y, Lin C, Reinhart B, Newell NR, Talavera-Rauh F, et al. Arabidopsis KANADI1 acts as a transcriptional repressor by interacting with a specific cis-element and regulates auxin biosynthesis, transport, and signaling in opposition to HD-ZIPIII factors. Plant Cell. 2014;26(1):246-62.

24. Ali MA, Abbas A, Kreil DP, Bohlmann H. Overexpression of the transcription factor RAP2.6 leads to enhanced callose deposition in syncytia and enhanced resistance against the beet cyst nematode Heterodera schachtii in Arabidopsis roots. BMC Plant Biol. 2013;13:47.

25. Doblin MS, Pettolino F, Bacic A. Plant cell walls: the skeleton of the plant world. Funct Plant Biol. 2010;37:357-81.

26. Miyakawa T, Fujita Y, Yamaguchi-Shinozaki K, Tanokura M. Structure and function of abscisic acid receptors. Trends Plant Sci. 2013;18(5):259-66.

27. Sreenivasulu N, Wobus U. Seed-development programs: a systems biology-based comparison between dicots and monocots. Annu Rev Plant Biol. 2013;64:189-217.

28. Zhu Q, Zhang J, Gao X, Tong J, Xiao L, Li W, et al. The Arabidopsis AP2/ARF transcription factor RAP2.6 participates in ABA, sat and osmotic stress responses. Gene. 2010;457:1-12.

29. Hedden P, Thomas SG. Gibberellin biosynthesis and its regulation. Biochem J. 2012:444(1):11-25.

30. Li J, Nie X, Tan JL, Berger F. Integration of epigenetic and genetic controls of seed size by cytokinin in Arabidopsis. Proc Natl Acad Sci U S A. 2013;110(38):15479-84.

31. Kudo T, Makita N, Kojima M, Tokunaga H, Sakakibara H. Cytokinin activity of cis-zeatin and phenotypic alterations induced by overexpression of putative cis-zeatin-O-glucosyltransferase in rice. Plant Physiol. 2012;160(1):319-31. 
32. Xia X, Zhang HM, Andriunas FA, Offler CE, Patrick JW. Extracellular hydrogen peroxide, produced through a respiratory burst oxidase/superoxide dismutase pathway, directs ingrowth wall formation in epidermal transfer cells of Vicia faba cotyledons. Plant Signal Behav. 2012;7(9):1125-8.

33. Fotopoulos V, Sanmartin M, Kanellis AK. Effect of ascorbate oxidase overexpression on ascorbate recycling gene expression in response to agents imposing oxidative stress. J Exp Bot. 2006;57(14):3933-43.

34. Yano H, Kuroda M. Disulfide proteome yields a detailed understanding of redox regulations: a model study of thioredoxin-linked reactions in seed germination. Proteomics. 2006;6(1):294-300.

35. Demidchik V, Maathius FJM. Physiological roles of non selective cation channels in plants: from salt stress to signaling and development. New Phytol. 2007;175(3):387-404.

36. Portillo M1, Cabrera J, Lindsey K, Topping J, Andrés MF, Emiliozzi M, et al. Distinct and conserved transcriptomic changes during nematode-induced giant cell development in tomato compared with Arabidopsis: a functional role for gene repression. New Phytol. 2013;197(4):1276-90.

37. Bulbert RA, Offler CE, McCurdy DW. Polarized microtubule deposition coincides with wall ingrowth formation in transfer cells of Vicia faba L. cotyledons. Protoplasma. 1998;201(1-2):8-16.

38. Tominaga $\mathrm{M}, \mathrm{Nakano}$ A. Plant-specific myosin $\mathrm{Xl}$, a molecular perspective. Front Plant Sci. 2012;3:211.

39. Hamada T, Ueda H, Kawase T, Hara-Nishimura I. Microtubules contribute to tubule elongation and anchoring of endoplasmic reticulum, resulting in high network complexity in Arabidopsis. Plant Physiol. 2014;166(4):1869-76.

40. Henty-Ridilla JL, Li J, Blanchoin L, Staiger CJ. Actin dynamics in the cortical array of plant cells. Curr Opin Plant Biol. 2013;16(6):678-87.

41. Zhang Y, Xiao Y, Du F, Cao L, Dong H, Ren H. Arabidopsis VILLIN4 is involved in root hair growth through regulating actin organization in a $\mathrm{Ca}^{2}$ +-dependent manner. New Phytol. 2011;190(3):667-82.

42. Hanton SL, Chatre L, Matheson LA, Rossi M, Held MA, Brandizzi F. Plant Sar1 isoforms with near-identical protein sequences exhibit different localisations and effects on secretion. Plant Mol Biol. 2008;67(3):283-94.

43. Xu J, Scheres B. Dissection of Arabidopsis ADP-RIBOSYLATION FACTOR 1 function in epidermal cell polarity. Plant Cell. 2005;17(2):525-36.

44. Zarsky V, Kulich I, Fendrych M, Pecenkova T. Exocyst complexes multiple functions in plant cells secretory pathways. Curr Opin Plant Biol. 2013;16(6):726-33.

45. Fujimoto M, Ueda T. Conserved and plant-unique mechanisms regulating plant post-Golgi traffic. FrontPlant Sci. 2012;3:197.

46. Konopka CA, Bednarek SY. Comparison of the dynamics and functional redundancy of the Arabidopsis dynamin-related isoforms DRP1A and DRP1C during plant development. Plant Physiol. 2008;147(4):1590-602.

47. Malinsky J, Opekarova M, Grossmann G, Tanner W. Membrane microdomains, rafts, and detergent-resistant membranes in plants and fungi. Annu Rev Plant Biol. 2013;64:501-29.

48. Nagano M, Ishikawa T, Ogawa Y, Iwabuchi M, Nakasone A, Shimamoto K, et al. Arabidopsis Bax inhibitor-1 promotes sphingolipid synthesis during cold stress by interacting with ceramide-modifying enzymes. Planta. 2014;240(1):77-89.

49. Markham JE, Molino D, Gissot L, Bellec Y, Hematy K, Marion J, et al. Sphingolipids containing very-long-chain fatty acids define a secretory pathway for specific polar plasma membrane protein targeting in Arabidopsis. Plant Cell. 2011;23(6):2362-78.

50. Haslam TM, Kunst L. Extending the story of very-long-chain fatty acid elongation. Plant Sci. 2013;210:93-107.

51. Saito K, Yonekura-Sakakibara K, Nakabayashi R, Higashi Y, Yamazaki M, Tohge T, et al. The flavonoid biosynthetic pathway in Arabidopsis: structural and genetic diversity. Plant Physiol Biochem. 2013;72:21-34.

52. Umate P. Oxysterol binding proteins (OSBPs) and their encoding genes in Arabidopsis and rice. Steroids. 2011;76(5):524-9.

53. Morisseau C. Role of epoxide hydrolases in lipid metabolism. Biochimie. 2013;95(1):91-5.

54. Rasbery JM, Shan H, LeClair RJ, Norman M, Matsuda SP, Bartel B. Arabidopsis thaliana squalene epoxidase 1 is essential for root and seed development. J Biol Chem. 2007;282(23):17002-13.

55. Vaughn KC, Talbot MJ, Offler CE, McCurdy DW. Wall ingrowths in epidermal transfer cells of Vicia faba cotyledons are modified primary walls marked by localized accumulations of arabinogalactan proteins. Plant Cell Physiol. 2007:48(1):159-68.
56. Doblin MS, Johnson KL, Humphries J, Newbigin EJ, Bacic A. Are designer plant cell walls a realistic aspiration or will the plasticity of the plant's metabolism win out? Curr Opin Biotechnol. 2014;26:108-14.

57. McFarlane HE, Doring A, Persson S. The cell biology of cellulose synthesis. Annu Rev Plant Biol. 2014;65:69-94.

58. Minic Z. Physiological roles of plant glycoside hydrolases. Planta. 2008;227(4):723-40.

59. Talbot MJ, Wasteneys GO, Offler CE, McCurdy DW. Cellulose synthesis is required for deposition of reticulate wall ingrowths in transfer cells. Plant Cell Physiol. 2007:48(1):147-58.

60. Rennie EA, Scheller HV. Xylan biosynthesis. Curr Opin Biotechnol. 2014;26:100-7.

61. Cleemput G, Hessing M, Van Oort M, Deconynck M, Delcour JA. Purification and characterization of a [beta]-D-xylosidase and an endo-xylanase from wheat flour. Plant Physiol. 1997;113(2):377-86.

62. Oikawa A, Lund CH, Sakuragi Y, Scheller HV. Golgi-localized enzyme complexes for plant cell wall biosynthesis. Trends Plant Sci. 2013;18(1):49-58.

63. Eklof JM, Brumer $\mathrm{H}$. The XTH gene family: an update on enzyme structure, function, and phylogeny in xyloglucan remodeling. Plant Physiol. 2010;153(2):456-66.

64. Sampedro J, Pardo B, Gianzo C, Guitian E, Revilla G, Zarra I. Lack of alphaxylosidase activity in Arabidopsis alters xyloglucan composition and results in growth defects. Plant Physiol. 2010;154(3):1105-15.

65. Wang L, Wang W, Wang YQ, Liu YY, Wang JX, Zhang XQ, et al. Arabidopsis galacturonosyltransferase (GAUT) 13 and GAUT14 have redundant functions in pollen tube growth. Mol Plant. 2013:6(4):1131-48.

66. Jolie RP, Duvetter T, Van Loey AM, Hendrickx ME. Pectin methylesterase and its proteinaceous inhibitor: a review. Carbohydr Res. 2010;345(18):2583-95.

67. Martin I, Hernandez-Nistal J, Albornos L, Labrador E, Dopico B. Betalll-Gal is involved in galactan reduction during phloem element differentiation in chickpea stems. Plant Cell Physiol. 2013;54(6):960-70.

68. De Storme N, Geelen D. Callose homeostasis at plasmodesmata: molecular regulators and developmental relevance. Front Plant Sci. 2014;5:138.

69. Thiel J, Müller M, Weschke W, Weber H. Amino acid metabolism at the maternal-filial boundary of young barley seeds: a microdissection-based study. Planta. 2009;230(1):205-13.

70. Wang J, Nayak S, Koch K, Ming R. Carbon partitioning in sugarcane (Saccharum species). Front Plant Sci. 2013;4:201.

71. Rocha S, Monjardino P, Mendonça D, Da Câmara Machado A, Fernandes R, Sampaio $\mathrm{P}$, et al. Lignification of developing maize (Zea mays L.) endosperm transfer cells and starchy endosperm cells. Front Plant Sci. 2014;20(5):102

72. Velasquez M, Salter JS, Dorosz JG, Petersen BL, Estevez JM. Recent advances on the posttranslational modifications of EXTs and their roles in plant cell walls. Front Plant Sci. 2012;3:93.

73. Gish LA, Clark SE. The RLK/Pelle family of kinases. Plant J. 2011;66(1):117-27.

74. Gucciardo S, Rathbun EA, Shanks M, Jenkyns S, Mak L, Durrant MC, et al. Epitope tagging of legume root nodule extensin modifies protein structure and crosslinking in cell walls of transformed tobacco leaves. Mol Plant Microbe Interact. 2005;18(1):24-32.

75. Wallace IS, Choi WG, Roberts DM. The structure, function and regulation of the nodulin 26-like intrinsic protein family of plant aquaglyceroporins. Biochim Biophys Acta. 2006;1758(8):1165-75.

76. Mansfield TA, Schultes NP, Mourad GS. AtAzg1 and AtAzg2 comprise a novel family of purine transporters in Arabidopsis. FEBS Lett. 2009;583(2):481-6.

77. Cornelius S, Traub M, Bernard C, Saizig C, Lang P, Möhimann T. Nucleoside transport across the plasma membrane mediated by equilibrative nucleoside transporter 3 influences metabolism of Arabidopsis seedlings. Plant Biology. 2012;14:696-705.

78. Remy E, Duque P. Beyond cellular detoxification: a plethora of physiological roles for MDR transporter homologs in plants. Front Physiol. 2014;5:201.

79. Wardini T, Talbot MJ, Offler CE, Patrick JW. Role of sugars in regulating transfer cell development in cotyledons of Vicia faba seeds. Protoplasma. 2007:230:75-88.

80. Silva-Sanchez C, Chen S, Zhu N, Li QB, Chourey PS. Proteomic comparison of basal endosperm in maize miniature1 mutant and its wild-type Mn1. Front Plant Sci. 2013;4:211. 